\title{
Analytics-Driven Networking: When the Computer becomes the Network
}

\author{
Inder Monga \\ Energy Sciences Network \\ Lawrence Berkeley National Lab, Berkeley CA \\ imonga@es.net
}

\begin{abstract}
As the era of 'human-managed networking' passes to 'analyticsdriven networking', more and more data about networks, including the constituent flows, is being tracked and retrieved. With networks now needing to be an effective sensor, new methods are being proposed to create and manage this streaming telemetry. While collection of this telemetry is happening at an unprecedented scale, it is unclear if the data is of enough resolution to make real-time decisions needed for fine-grained control, or for the application of new machine learning/artificial intelligence techniques. New techniques are being developed to provide high-precision telemetry as new analytics to take advantage of that are being developed.

While telemetry is being used to provide insights about networks, applications want more instrumentation from the currently opaque network. Currently, there is little ability for the applications to interact with the network to exchange information, negotiate performance parameters, discover desired performance metrics, or receive status/troubleshooting information in real time.

This talk will share challenges, opportunities and new research in progress, from a science network view, as we evolve the network to an analytics-driven, autonomous system. In addition, the talk will discuss the new nation-scale testbeds being built, with new capabilities, like in-network processing, that could revolutionize how we measure and process end-to-end workflows.
\end{abstract}

\section{CCS Concepts/ACM Classifiers}

- Programmable networks, Network monitoring, in-network processing, network management, network measurement, network experimentation, networking design principles

\section{Author Keywords}

Network telemetry; precision telemetry; network testbeds; data analytics; autonomic networks;

Permission to make digital or hard copies of part or all of this work for personal or classroom use is granted without fee provided that copies are not made or distributed for profit or commercial advantage and that copies bear this notice and the full citation on the first page. Copyrights for third-party components of this work must be honored. For all other uses, contact the Owner/Author.

SNTA '20, fune 23-26, 2020, Stockholm, Sweden.

(c) 2019 Copyright is held by the owner/author(s)

ACM ISBN 978-1-4503-7980-9/20/06.

https://doi.org/10.1145/3391812.3396266

\section{BIOGRAPHY}

Indermohan (Inder) S. Monga serves as the Division Director for Scientific Networking Division, Lawrence Berkeley National Lab and Executive Director of Energy Sciences Network, a highperformance network user facility optimized for large-scale science, interconnecting the National Laboratory System in the United States. Under his leadership, the organization focuses on advancing the science of networking for collaborative and distributed research applications. He contributes to ongoing research projects tackling network programmability, analytics and quality of experience driving convergence between application layer and the network.

He currently holds 23 patents and has $20+$ years of industry and research experience in telecommunications and data networking. His work experience in the private sector includes network engineering for Wellfleet Communications and Nortel where he focused on application and network convergence. His undergraduate degree is in electrical/electronics engineering from Indian Institute of Technology in Kanpur, India, with graduate studies from Boston University.

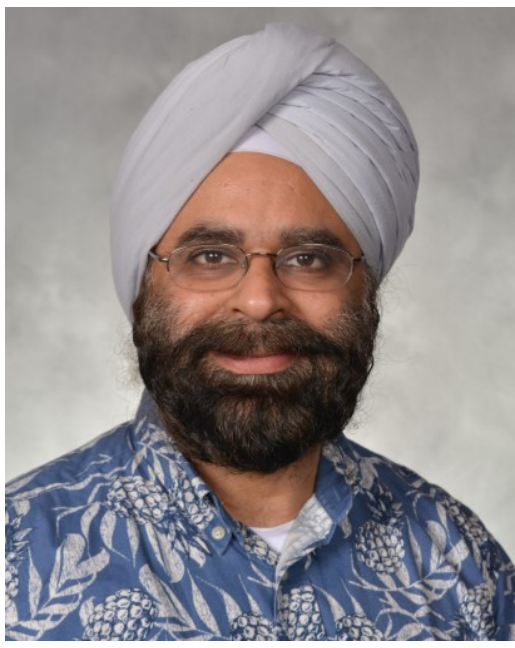

